\title{
Comparison of Random Error Analysis Methods for Fiber Optic Gyro Based on Allan Variance
}

\section{Du Xiao Jing, Zeng Chun, Li Huai Jian}

School of Aerospace Engineering, Beijing Institute of Technology, Beijing, China

\section{Email address:}

duxiaojing2633@163.com (Du Xiao Jing), Spring7974@163.com (Zeng Chun), lihuaijian@bit.edu.cn (Li Huai Jian)

\section{To cite this article:}

Du Xiao Jing, Zeng Chun, Li Huai Jian. Comparison of Random Error Analysis Methods for Fiber Optic Gyro Based on Allan Variance. Science Discovery. Vol. 5, No. 5, 2017, pp. 375-379. doi: 10.11648/j.sd.20170505.22

Received: June 23, 2017; Accepted: August 16, 2017; Published: September 14, 2017

\begin{abstract}
It is an effective method to improve the performance of inertial instrument and the accuracy of inertial navigation system by analyzing the random drift of FOG (Fiber Optic Gyro). Allan variance FOG random error analysis method, through analyze and compare Allan variance of the original data, you can correctly evaluate the gyroscope performance indicators. In this paper, we introduce the several different random error analysis method of FOG based on Allan variance, and analyze the different Allan variance of the same group by writing the program, and more, compare the three common quantities of the FOG random error model. The results show that the modified Allan variance method obviously takes a lot of time in the processing time, but the " $2^{k "}$ Allan variance law is calculated more quickly. In the processing effect, the modified Allan variance method can get better calculation results. In general, the " $k$ " Allan variance method and the smooth Allan variance method are more balanced in the computational time and the computational effect. Several Allan variance methods can effectively identify the random error components of FOG, in the actual situation, according to different conditions of use to choose the appropriate treatment.
\end{abstract}

Keywords: Fiber Optic Gyro, Random Error Model, Allan Variance Eethod

\section{基于Allan方差光纤陀螺随机误差分析方法比较}

杜小菁, 曾春, 李怀建

宇航学院, 北京理工大学, 北京, 中国

邮箱

duxiaojing2633@163.com（杜小等）, Spring7974@163.com（曾春）, lihuaijian@bit.edu.cn（李怀建）

摘要: 对光纤陀螺随机漂移进行分析研究是改进惯性仪器本身性能、提高惯导系统精度的有效方法。Allan方差光纤陀 螺随机误差的分析方法，通过对原始数据的Allan方差分析对比，可以正确评价陀螺仪的性能指标。本文分别介绍了基 于Allan方差的几种不同光纤陀螺随机误差分析方法, 通过编写程序对同一组数进行不同Allan方差分析, 对比光纤陀螺 随机误差模型的 3 个常见量。结果表明：在处理时间上，经过修正的Allan方差法明显需要大量的时间，“ $2^{k}$ ” Allan方差 法则计算比较迅速; 在处理效果上，经过修正的Allan方差法可以获得较好的计算效果; 一般情况下，“ $k$ ” Allan方差法 以及平滑Allan方差法在计算时间以及计算效果上比较均衡。几种Allan方差法都可以有效地辨识光纤陀螺的各项随机误 差成分, 在实际情况中可以根据不同的使用条件选择合适的处理方法。

关键词: 光纤陀螺, 随机误差模型, Allan方差法 


\section{1. 引言}

光纤陀螺漂移的一个分量是随机漂移。随机漂移是衡量 陀螺精度的最重要指标, 它随时间变化且对惯导系统精度产 生很大影响。分析光纤陀螺随机误差模型有利于对其进行正 确的建模。Allan方差[1]分析法是分析随机误差的重要方法, 基于Allan方差思想, 人们在使用Allan方差时有如下几种演 变的Allan方差法: “ $k$ ” Allan方差法 $[2,3]$ 、 “ $2^{k}$ ” Allan方差 法 $[4,5,6]$ 、平滑Allan方差法 [7,8]以及改进Allan方差法 $[9$, 10]。本文分别介绍这几种方法, 然后对静止条件下的光纤 陀螺输出值进行分别处理, 对比处理数据所消耗的时间以及 处理效果, 分析比较了几种Allan方差处理方法的差异, 对研 究或者使用Allan方差的人们有不错的借鉴意义。

\section{2. 光纤陀螺随机误差模型分析}

光纤陀螺的随机误差主要包括量化噪声（QN）、角 度随机游走 (ARW) 、零偏不稳定性 (BI) 、角速率随 机游走（RRW）、速率斜坡（RR）5种噪声[11]。

1) 量化噪声: 由光纤陀螺的数字特性引起, 代表了 光纤陀螺的最低分辨率。量化噪声的Allan方差为式 (1), 式中 $Q$ 为量化噪声系数。

$$
\sigma_{Q N}^{2}(\tau)=\frac{3 Q^{2}}{\tau^{2}}
$$

2) 角度随机游走系数: 主要来源于光子的自发辐射、 探测器的散粒噪声以及其他相关时间比采样周期短的高 频噪声。角度随机游走的Allan方差为式 (2), 式中 $N$ 为 角度随机游走系数。

$$
\sigma_{A R W}^{2}(\tau)=\frac{N^{2}}{\tau}
$$

3) 零偏不稳定性: 主要来源于光纤陀螺中的放电组 件噪声、环境噪声, 以及产生随机闪秌的组件。零偏不稳 定性噪声的Allan方差为式 (3), 式中 $B$ 为零偏不稳定性 系数。

$$
\sigma_{B I}^{2}(\tau)=\frac{B^{2} 2 \ln 2}{\pi}
$$

4) 角速率随机游走: 一种没有确定来源的随机过程, 当指数互相关过程的互相关时间很长时可表现为此类噪 声。角速率随机游走的Allan方差为式 (4), 式中 $K$ 为角 速率随机游走系数。

$$
\sigma_{R R W}^{2}(\tau)=\frac{K^{2} \tau}{3}
$$

5 ）速率斜坡: 由于光纤陀螺的光源强度呈极慢的单 调变化, 并持续很长时间而表现出来的一种噪声。也可能 是平台保持非常小的加速度进而表现为光纤陀螺的真实
输入。速率斜坡的Allan方差为式 (5), 式中 $R$ 为速率斜 坡系数。

$$
\sigma_{R R}^{2}(\tau)=\frac{R^{2} \tau^{2}}{2}
$$

假设各噪声源统计独立, 则计算得到的Allan方差是各 类型误差的平方和。即

$$
\begin{gathered}
\sigma_{A}^{2}(\tau)=\sigma_{Q N}^{2}(\tau)+\sigma_{A R W}^{2}(\tau)+ \\
\sigma_{B I}^{2}(\tau)+\sigma_{R R W}^{2}(\tau)+\sigma_{R R}^{2}(\tau)
\end{gathered}
$$

式中各误差项可表示为正比于相关时间 $\tau$ 的 $-2 \sim+2$ 次幂的系数表达式, 所以式（6）可以写成:

$$
\sigma_{A}^{2}(\tau)=\sum_{i=-2}^{2} A_{i}^{2} \tau^{i}
$$

可以得到光纤陀螺各项随机误差如表1:

表1 Allan方差对数图误差特性。

\begin{tabular}{llll}
\hline 噪声类型 & Allan方差 & 参数 & 曲线坡度 \\
\hline 量化噪声 & $\sigma^{2}(\tau)=3(\mathrm{Q} / \tau)^{2}$ & $Q$ & -1 \\
角度随机游走 & $\sigma^{2}(\tau)=N^{2} / \tau$ & $N$ & $-1 / 2$ \\
零偏不稳定性 & $\sigma^{2}(\tau)=(B / f)^{2}$ & $B$ & 0 \\
速率随机游走 & $\sigma^{2}(\tau)=K^{2} \tau / 3$ & $K$ & $1 / 2$ \\
速率斜坡 & $\sigma^{2}(\tau)=(R \tau)^{2} / 2$ & $R$ & 1 \\
\hline
\end{tabular}

\section{Allan方差理论}

Allan方差是在时域上对频域特性进行分析的一种方 法, 最早由美国国家标准局的David Allan提出, 是IEEE 公认的陀螺仪参数辨识的标准方法。通过该方法, 可以对 陀螺输出数据构成一个样本空间进行处理, 从而辨识出陀 螺仪各项误差的参数（表1）。

\section{1. 经典Allan方差理论}

\subsection{1. “ $k ” \mathrm{~A} 11 \mathrm{an}$ 方差原理}

1) 采样得到间隔时间为 $t_{0}$ 的 $n$ 个连续初始样本 $\Omega_{i}(i=1, \cdots, n)$ 。

2) 、对 $n$ 个初始采样样本进行图1所示的二次采样, 采样时间取 $\tau=k t_{0}$, 分别取 $\tau$ 等于 $t_{0}, 2 t_{0}, \cdots, k t_{0}$, $k=1,2,3,4 \cdots$, 其中 $k<n / 2$, 则组数 $m=n / k$, 每组包 含 $k$ 个采样点, 所有陀螺输出角速率可以表示为:

$$
\underbrace{\omega_{1}, \omega_{2}, \cdots, \omega_{k}}_{i=1}, \cdots, \underbrace{\omega_{n-k+1}, \omega_{n-k+2}, \cdots, \omega_{n-k+k}}_{i=m}
$$




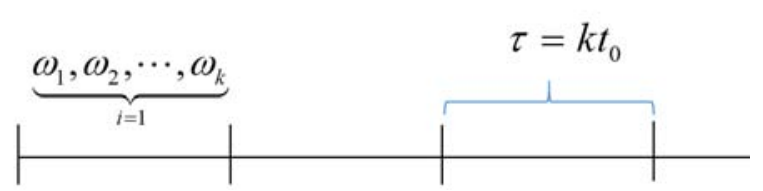

图1 “ $k$ ” Allan方差二次采样示意图。

求出每一时间长度 $\tau$ 的数组平均值为:

$$
\bar{\Omega}_{p}(\tau)=\frac{1}{k} \sum_{i=1}^{k} \Omega_{(j-1) k+i}\left(t_{0}\right) \quad j=1, \cdots, m
$$

3) 求相邻两个数组平均的差, 见式 (10)

$$
\xi_{p+1, p} \equiv \bar{\Omega}_{p+1}(\tau)-\bar{\Omega}_{p}(\tau)
$$

给定 $\tau$, 上式定义了一个元素为数组平均之差的随机 变量集合 $\left\{\xi_{p+1, p}, p=1, \wedge \wedge, \mathrm{m}-1\right\}$, 共有 $m-1$ 个这样的数组 平均的差。

4) 求随机变量集合 $\left\{\xi_{p+1, p}, p=1, \wedge \wedge, m-1\right\}$ 的方差, 见式 (11) - (12) :

$$
\sigma^{2}(\tau)=\frac{1}{2(m-2)} \sum_{p=1}^{m-2}\left[\xi_{p+2, p+1}-\xi_{p+1, p}\right]^{2}
$$

即:

$$
\sigma^{2}(\tau)=\frac{1}{2(m-2)} \sum_{p=1}^{m-2}\left[\bar{\Omega}_{p+2}(\tau)-2 \bar{\Omega}_{p+1}(\tau)+\bar{\Omega}_{p}(\tau)\right]^{2}
$$

5 ) 分别取不同的 $\tau$, 重复上述过程, 在双对数坐标 系中得到一个 $\sigma(\tau) \sim \tau$ 曲线

\section{1.2. “ $2^{k} ” \mathrm{~A} 11 \mathrm{an}$ 方差原理}

本方法在原理上与 2.1.1的步骤基本相同, 唯一的区别 在 $k$ 的取值上。“ $2^{k}$ ” Allan方差原理中 $k$ 取:

$$
k=2^{0}, 2^{1}, 2^{2}, 2^{3}, \cdots 2^{x} .(k<n / 2)
$$

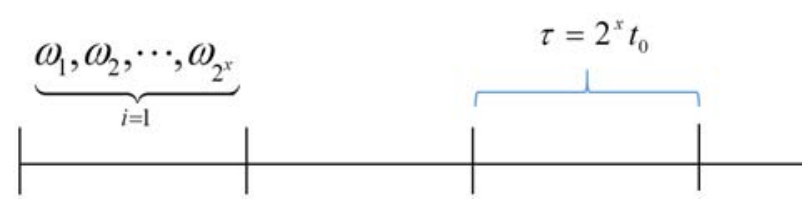

图2 “ $2^{k}$ ” Allan方差二次采样示意图。

\section{2. 基于平滑的Allan方差方法}

如图3所示，所谓平滑采样即在采样的时候以“1个元 素”为间隔，后一组在前一组的基础上去掉第一个元素而 加上一个新的元素。

1）同3.1.1节的1）。
2) 对这 $n$ 个初始样本进行图3所示的二次采样, 采样 时间 $\tau=k t_{0}$, 分别取 $\tau$ 等于 $t_{0}, 2 t_{0}, \cdots, k t_{0}(k<n / 2)$, 每组 包含 $k$ 个采样点, 求出每一个时间长度 $\tau$ 的数组平均值 (数组平均), 共有 $m=n-k+1$ 个这样的数组平均。见 式（13）。

$$
\begin{gathered}
\bar{\Omega}_{p}(\tau)=\frac{1}{k} \sum_{i=p}^{p+k-1} \Omega_{i}\left(t_{0}\right) \\
p=1,2, \cdots \cdots, n-k+1
\end{gathered}
$$

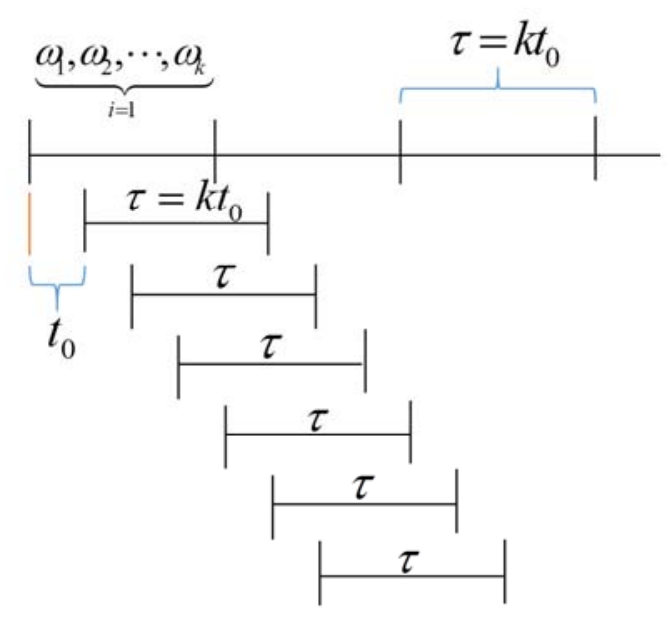

图3 基于平滑的Allan方差二次采样示意图。

3 ) 求相邻两个数组平均的差, 见式 (10) 给定 $\tau$, 上式定义了一个元素为数组平均之差的随机变量集合 $\left\{\xi_{p+1, p}, p=1, \wedge \wedge, n-k\right\}$, 共有 $n-k$ 个这样的数组平均的差。

4 ) 求方差, 随机变量的集合为 $\left\{\xi_{p+1, p}, p=1, \wedge \wedge, n-k\right\}$, 其方差见式（14）-（15）:

$$
\sigma^{2}(\tau)=\frac{1}{2(\mathrm{n}-\mathrm{k}-1)} \sum_{p=1}^{n-k-1}\left[\xi_{p+2, p+1}-\xi_{p+1, p}\right]^{2}
$$

即:

$$
\begin{array}{r}
\sigma^{2}(\tau)=\frac{1}{2(\mathrm{n}-\mathrm{k}-1)} \sum_{p=1}^{n-k-1}\left[\bar{\Omega}_{p+2}(\tau)\right. \\
\left.-2 \bar{\Omega}_{p+1}(\tau)+\bar{\Omega}_{p}(\tau)\right]^{2}
\end{array}
$$

5）、同3.1.1步骤5）。

\section{3. 改进A11an方差}

改进Allan方差与基于平滑的Allan方差在1）、2）步 骤都相同, 不同之处在数组平均差的求法。改进Allan方差 具体计算步骤如下:

1）、2）与 2.2 节的步骤一样。 
3) 求间隔为 $k$ 的数组平均的差, 见式 (10) 给定 $\tau$, 上式定义了一个元素为数组平均之差的随机变量集合 $\left\{\xi_{p+k, p}, p=1, \wedge \wedge, n-2 k\right\}$, 共有 $n-2 k$ 个这样的数组平均 的差。

4) 改进方差求法:

$$
\begin{aligned}
\operatorname{Mod}^{2}(\tau) & =\frac{1}{2 \tau^{2} k^{2}(\mathrm{n}-3 k-1)} \sum_{j=1}^{\mathrm{n}-3 k-1} \\
& {\left[\sum_{i=j}^{k+j-1}\left(\Omega_{i+2 k}-2 \Omega_{i+k}+\Omega_{i}\right)\right]^{2} }
\end{aligned}
$$

5) 分别取不同的 $\tau$, 重复上述过程, 在双对数坐标 系中得到一个 $\operatorname{Mod} \sigma(\tau) \sim \tau$ 曲线。

\section{4. 仿真比较}

由于受改进Allan方差处理数据能力限制, 本仿真在静 止条件下, 用某型光纤陀螺采集3016个陀螺仪输出值, 如 图4所示:

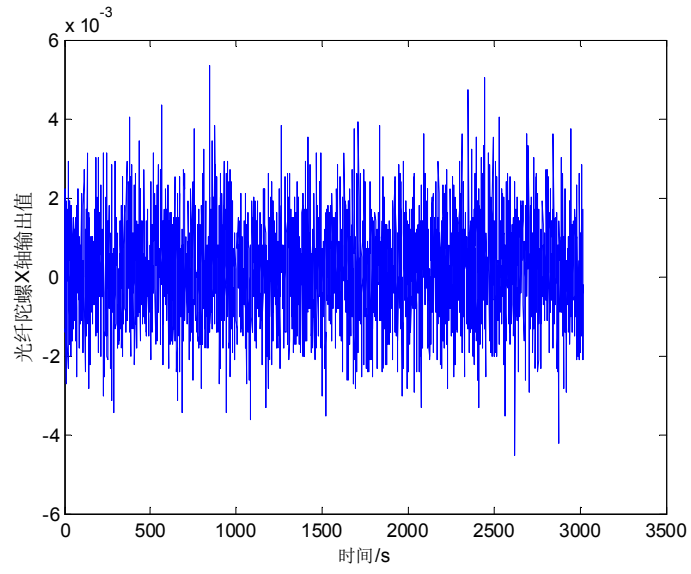

图4 静止条件下光纤陀螺X轴向输出值。

\subsection{1. 处理时间分析}

在处理器为酷睿 $17-6700$ 、内存为 $8 \mathrm{~GB}$ 的64位Windows 7专业版电脑上安装 Qt5.7, 用Qt编写“ $k$ ” Allan方差法、 “ $2^{k}$ ” Allan方差法、平滑Allan方差法以及改进Allan方差法 的相应求解程序, 运行程序并记录核心算法所需时间如表 2所示:

表2 几种处理方法所需时间比较。

\begin{tabular}{ll}
\hline 核心算法运行时间 & 所需时间 $/ \mathbf{S}$ \\
\hline “ $k$ Allan方差法 & 0.119 \\
“ $2^{k}$ ” Allan方差法 & 0.002 \\
平滑Allan方差法 & 0.611 \\
基于平滑改进Allan方差法 & 1223.21 \\
\hline
\end{tabular}

从表2可以得出, “ $2^{k}$ ” Allan方差法的处理速度最快, 而改进Allan方差法的处理速度最慢, 在量级上这两种方法相 差非常大; “ $k$ ” Allan方差法相比平滑Allan方差法处理速度更快一些, 在量级上这两种方法一样。所以, 当样本数量非 常非常大时, “ $2^{k} ”$ Allan方差法比较适合; 而改进Allan方差法只适合处理小样本数据。

\subsection{2. 精度分析}

分别利用上面所提的几种方法对这采集数据进行Allan方差求解，得到表3：表3 噪声源误差系数 $10^{-5}$ 。

\begin{tabular}{lllll}
\hline 噪声名称 & “ $\boldsymbol{k}$ ” Allan方差法 & $\cdots 2^{k}$ ” Allan方差法 & 平滑Allan方差法 & 基于平滑改进Allan方差法 \\
\hline 量化噪声 $Q$ & 12.9363 & 12.0997 & 12.1945 & 1.32604 \\
角度随机游走 $N$ & 67.3074 & 67.6563 & 56.9338 & 6.6833 \\
零偏不稳定性系数 $B$ & $8.74045 \mathrm{e}-3$ & $-1.76063 \mathrm{e}-3$ & $2.70277 \mathrm{e}-4$ & $1.24934 \mathrm{e}-5$ \\
角速率随机游走 $K$ & - & - & - & - \\
速率斜坡 $R$ & - & - & - & - \\
\hline
\end{tabular}

\section{从表 2 中的数据可以看出:}

1、受采集样本容量的限制, 角速率随机游走 $K$ 和速 率斜坡 $R$ 没能求解出来，这是由于角速率随机游走 $K$ 和 速率斜坡 $R$ 存在于低频段，而本测试样本小，所以无法测 出低频段数据。

2、“ $k$ ” Allan方差法、“ $2^{k}$ ” Allan方差法和平滑Allan 方差法处理得到的量化噪声、角度随机游走系数在量级上 是一样, 但是相对来说平滑Allan方差法处理效果更好。而 相对于改进Allan方差法, 改进Allan方差法的处理效果明 显优于其他三种方法。
3、通过对比角度随机游走系数与零偏不稳定性系数, 平滑Allan方差法处理效果优于 “ $k$ ” Allan方差法与 “ $2^{k}$ ” Allan方差法但是却没有改进Allan方差法好。

通过上面的对比分析, 改进Allan方差法明显优于其他 三种方法, 相比之下平滑Allan方差法又比“ $k$ ” Allan方差 法与“ $2^{k}$ ” Allan方差法处理效果好。

\section{4. 结论}

Allan方差法能够较好的辨析出光纤陀螺的量化噪声、 随机游走系数与零偏不稳定性, 而角速率随机游走与速率 斜坡由于体现于较低的采样频率, 所以小容量数据无法得 
到。不同的Allan方差处理方法分析出的误差精度不同, 所 需的处理时间也存在较大的差异。虽然改进的Allan方差法 相对于其他几种在误差精度上较好, 但是其明显需要大量 的计算时间作为代价, 特别是在大样本情况下, 或者需要 实时分析下, 改进的Allan方差法不适合。相对而言的, 在 大样本及实时性的要求下, “ $k^{2}$ ” Allan方差法比较适合。 当然，除了这些特殊条件，平滑Allan方差法相对于“ $k$ ” Allan方差法在处理精度上有一定优势

\section{参考文献}

[1] Howe D A, Allan D W, Barnes J A. Properties of signal sources and measurement methods $[\mathrm{C}] / /$ Thirty Fifth Annual Frequency Control Symposium 1981, 27-29 May 1981: Electron. Ind. Assoc, 1981. 1-47.

[2] 金毅, 吴训忠, 谢聂. 基于Allan方差的光纤陀螺随机漂移 建模与仿真 $[\mathrm{J}]$. 应用光学, 2014,(03): 547-551。

[3] 米月琴, 李汉舟. 基于Allan方差的典型应用 [J]. 机电技术, 2016, (01):20-22。
[4] 宋凝芳, 张中刚, 李立京, 金靖. 光纤陀螺随机游走系数 的分析研究 [J]. 中国惯性技术学报, 2004,(04): 36-40。

[5] L H. The fiber optic gyroscope [J]. London: Artech House, 1993.

[6] IEEE Std 952-1997. IEEE Standard Specification Format Guide and Test Procedure for Single Axis Interferometric Fiber Optic Gyros [J]. 1997.

[7] GJB-2426-95. 光纤陀螺仪测试方法[J]. 1995。

[8] GJB 2426A-2004. 光纤陀螺仪测试方法[J]. 2004。

[9] 祝树生, 任建新, 张安峰. 光纤陀螺随机游走分析方法研 究[J]. 应用光学, 2009,(06):1003-1006。

[10] Allan D W, Barnes J A. A modified 'Allan variance' with increased oscillator characterization ability $[\mathrm{C}] / /$ Thirty Fifth Annual Frequency Control Symposium 1981, 27-29 May 1981: Electron. Ind. Assoc., 1981. 470-475.

[11] 张谦, 王玮, 王蕾, 高鹏宇. 基于动态Allan方差的光纤陀 螺随机误差分析及算法改进 [J]. 光学学报, 2015,(04): 65-71。 